\title{
PSICOLOGÍA PARA UN MUNDO DE DESIGUALDADES, DOMINACIÓN Y MACHISMO
}

\author{
Francisco Humberto Eduardo Almeida Acosta ${ }^{1}$ \\ Universidad Iberoamericana Puebla, México
}

\section{RESUMEN}

Nuestras inquietudes como psicólogos se centran en procesos mentales y conductuales, que han sido los mismos en culturas orientales, africanas y amerindias. Pero el origen de nuestra "ciencia", lo situamos en lo razonable y en lo masculino. Hemos descuidado lo sabio, lo vital y lo femenino. Propongo la búsqueda de una psicología más universal y más relevante.Si quisiéramos una psicología para la inclusión de todos los humanos, tendríamos que enfocar nuestros afanes hacia otros horizontes, hacia un mundo de lo común y de lo solidario. Desde mi experiencia en comunidades puedo afirmar que los pueblos indígenas tienen una respuesta. Ahora o nunca es su tiempo. Como psicólogos podemos organizar nuestras vidas con actos de resistencia y fraternidad contra los poderes que mantienen al capitalismo, a la colonialidad y al machismo.

\section{Palabras clave}

psicología, comunidad, dominación, machismo, solidaridad

\section{RÉSUMÉ}

Nos inquiétudes en tant que psychologues se trouvent concentrées dans les mêmes processus mentaux et comportementaux que ceux des cultures orientales, africaines et amerindiennes. Mais nous situons l'origine de notre "science" sur le raisonnement et le masculine. Nous avons négligé la sagesse, la vitalité et le féminin. Je propose la recherche d'une psychologie plus universelle.Si nous voudrions developper une Psychologie plus inclusive pour tous les humains nous devrions diriger nos efforts vers d'autres horizons, vers un monde du commun et du solidaire. A partir de mes travaux en communautés je puis affirmer que les peuples indigènes offrent des approches en ce sens. Maintenant le temps est arrivé de les prendre en compte.En tant que psychologues nous pouvons organiser nos vies vers la résistence et la fraternité, et contre les pouvoirs que favorisent le capitalisme, la colonialité et le machisme.

\section{Mots clés}

Psychologie, communauté, domination, machisme, solidarité

\footnotetext{
${ }^{1}$ Correspondence about this article shpould be addressed to Francisco Humberto Almeida Acosta. Email: eduardo.almeida@iberopuebla.mx
} 


\section{PSYCHOLOGY FOR A WORLD OF INEQUALITIES, DOMINATION AND MACHISMO}

Es urgente abordar nuestras inquietudes como psicólogos y científicos desde perspectivas que provisionalmente resumo con el título de "Una psicología crítica para el buen vivir en Nuestra América y en Canadá y en Estados Unidos".

Un pensamiento complaciente, adormecedor no permite la búsqueda de sociedades más justas. No lo permite la ausencia de un pensamiento crítico y desestabilizador que vaya más allá de lo intrapsíquico y lo individual. Cf. el número 1, vol. 74 del American Psychologist.

Una mirada neoliberal del sujeto, individualista, consumista, corporativista, capitalista con sus obsesiones de excelencia, plenitud, talento, alto rendimiento, no es apta para abatir las brechas de la desigualdad. La inmensa mayoría del planeta la forman los desplazados, los pobres, los explotados, los excluidos. Cf. las orientaciones del XXVI Congreso Interamericano de Psicología (julio 22 al 27 de 2017).

Posturas institucionales clasistas, discriminatorias, coloniales, racistas, dependientes no sirven para diseñar estrategias contra la expulsión social y la absurda desigualdad. El mundo no es sólo el de habla inglesa, no es sólo el de los centros de poder y de derroche. Cf. enfoques recientes sobre Reforma Educativa de la OCDE (2019).

Enfoques desarrollistas, paternalistas, legalistas, conservadores, patriarcales, se oponen a un abordaje crítico de la comunidad, la sexualidad, la política, el derecho y el medio ambiente. El mundo de la vida, el de la realidad humana va bastante más allá de los enclaves de los privilegiados y de las supuestas clases medias. Cf. las posturas del neoliberalismo académico de la IUPsyS.

Como propusieron los organizadores del Primer Congreso Panafricano de Psicología (2017) que se celebró en Durban, Sudáfrica en el mes de septiembre de 2017, se requiere de una "Psicología para la sociedad", una psicología para todo el mundo.

\section{Psicología y psicólogos para la sociedad}

La psicología, y en particular la psicología social, quedaron al margen del pensamiento crítico que se desarrolló en las Ciencias Sociales y Humanas desde los años 30 del siglo pasado. Se quedaron aisladas de los notables desarrollos que fueron generando el pensamiento anticapitalista, descolonial y despatriarcalizante (Rodríguez, 2014). No pudieron estar atentas a la evolución de nuestras sociedades explotadas, despreciadas y violadas en múltiples ocasiones por los centros de poder hegemónico. Frente a nuestras existencias sitiadas (Subirats, 2007) no han cesado de aparecer y de activarse experiencias de lucha abiertas y clandestinas que han mantenido vigente y alerta la posibilidad y la esperanza de otros modos de vida para nuestras maltratadas, explotadas y despreciadas poblaciones. La llegada de Donald Trump a la presidencia de Estados Unidos ha hecho muy patentes el racismo, la xenofobia y la misoginia de una numerosa población de ese país y de las élites mimetizadas de muchos Estados que miran hacia los países ricos en búsqueda del "bien estar". Es cierto que ha habido aperturas de la psicología social hacia una perspectiva crítica (el libro "Decolonialidad desde la Psicología Social Comunitaria", 2018, de Germán Rozas) pero no es lo más general.

No se ha problematizado a niveles de fecundidad teórica y práctica las acciones y omisiones de los psicólogos frente a tanta violencia que sufren actualmente los niños, las mujeres, los migrantes, los desplazados, las víctimas del crimen organizado y de las guerras de poder entre naciones arrogantes y ambiciosas. No se ha problematizado la ausencia de la psicología en la dinámica central de nuestros países, la de la vinculación entre el Estado, las corporaciones trasnacionales y el crimen organizado como lo reveló de manera emblemática la desaparición de los 43 estudiantes de Ayotzinapa.

El estudio del machismo en las Ciencias Sociales se intensificó cuando en 1949 Simone de Bauvoir publicó "Le deuximème sex" (De Bauvoir, 2013). Ya entonces ella se refería a la opresión 
que viven las mujeres. Es importante que la psicología se haya dado cuenta, aunque tardíamente, de la necesidad de una ruptura más concreta y más consciente con el patriarcado. Ello forma parte del proceso de liberación para romper la enajenación como ha dicho Martín-Baró (1998). Esto implica la lucha para destruir el modelo patriarcal, racista y capitalista (Pisano, 2001). Al comentar el libro "Más allá del feminismo: Caminos para andar" que coordinó Márgara Millán en 2014, María Eugenia Sánchez (2015) expresó: "El libro... es un diálogo... que parte desde lo femenino, desde lo que ha sido invisibilizado, oprimido, expoliado, exiliado... La emancipación sería la reconfiguración de relaciones, asumiendo la tensión y la conflictividad en su interacción... donde precisamente emerge una creatividad generativa". Es un libro que propone una epistemología rebelde. Que puede llevar al "parlamento de los cuerpos" que dice María Galindo (s.f.) "lleva a dos críticas centrales a las democracias: los parlamentos sin democracia y las democracias sin cuerpo".

¿No es verdad que seguimos enfrascados en atender con prioridad problemas orientados a la búsqueda de la excelencia, la "calidad de vida", el éxito a como dé lugar, el "alto rendimiento", la plenitud existencial personal a expensas de la dignidad de la mayoría de la población mundial?

Como seres humanos, y sin olvidar las dificultades y problemas que implica toda vida humana, los psicólogos hemos sido privilegiados por disfrutar de la salud necesaria para llegar a haber hecho estudios; por haber podido llegar a ser profesionales en sociedades muy dañadas por todo tipo de injusticias.

Como profesionistas la dignidad de nuestro trabajo radica en el servicio para la buena vida de nuestras comunidades y de nuestras sociedades. Como profesionales de la psicología constituimos un cuerpo de servicio con una gran potencialidad para contribuir al "buen vivir" de nuestros congéneres (Martín-Baro, 1976; Ruiz, comunicación personal, 2009; Tutu, comunicación personal, 2012). Los que nos dedicamos al trabajo académico vivimos insertos en instituciones que pueden llegar a ser centros de reflexividad para atender a las situaciones adversas que comportan los cambios de época que estamos viviendo. Nos ocupamos en producir, valorar, criticar y compartir el conocimiento psicológico. Este conocimiento es indispensable para la buena vida de las poblaciones, para fomentar la resiliencia, la autoestima, la actitud positiva y fecunda frente a la adversidad. Como científicos, con una aproximación no sectaria a lo que es "ciencia" -porque la realidad está demandando nuevos cortes del conocimiento- tenemos que considerar que la ciencia psicológica se constituye con saberes académicos de laboratorio, de biblioteca, de internet, de cuerpos académicos, pero también con saberes de acción y saberes de vida que se producen en el trabajo, en la interacción con las personas con las que trabajamos, y en la experiencia vital que se transmite entre las generaciones. Lo que sabemos en psicología proviene de todas esas fuentes. Esos saberes y prácticas sobre la vida humana se refieren a procesos mentales y conductuales, observables e interpretables, personales y colectivos.

Quisiera subrayar que en todo esto nuestros cuerpos, alimentados o desnutridos, protegidos o desamparados, sanos o enfermos, valorados o despreciados, tienen todo que ver con nuestra atención y percepción, motivación y emoción, pensamiento y lenguaje, memoria y aprendizaje. Esto parecemos olvidarlo cuando nos despreocupamos por las situaciones de injusticia, abuso, privación, angustia y miedo que padece la mayoría de la población de Nuestra América. Aquí me refiero a la relación salud-psicología y a las deficiencias de nuestras poblaciones.

Fácilmente olvidamos las fecundas advertencias que hizo Edgar Morín (2000) con relación a los saberes y que también podemos aplicar a la psicología ¿Está nuestro conocimiento exento de ilusiones y errores? ¿Nuestro conocimiento es pertinente para el contexto en el que nos desenvolvemos? ¿Olvidamos que todos participamos de la misma condición humana y de que todos nuestros destinos son compartidos? ¿No perdemos de vista que para entender a los humanos los lazos sociales son primordiales? ¿Somos conscientes de que la incertidumbre es la realidad de todos nosotros, de que tenemos que organizarnos para enfrentar riesgos y diseñar estrategias para la vida? ¿No hemos desviado la mirada (Klein, 2014) ante el cambio climático y la realidad de que vivimos 
ya en una era planetaria más allá de los neoliberalismos? ¿Hemos tratado de captar que la gran carencia de la que sufrimos hoy los humanos es la falta de compasión? Estas preguntas son reflexiones a partir de las inquietudes de este autor, uno de los pensadores más agudos de nuestro tiempo.

La ciencia psicológica, como se visualiza en la gran mayoría de nuestras instituciones, como la mayoría de las Ciencias Sociales y Humanas que manejamos actualmente, proviene de la burguesía del siglo XIX (Martín-Baro, 1976). Si nos apartamos de esa concepción estrecha de "Ciencia Psicológica" no tardaremos en descubrir que la comprensión de la psicología humana es un saber bastante más antiguo que ésa, que es una sabiduría ancestral que se ha ido creando a lo largo de la historia humana en todas las latitudes. El cuerpo de saberes sobre mente y conducta antes del cientificismo lo podemos encontrar en todos los continentes, con diferentes nombres y haciendo hincapié en variadas aproximaciones de sabiduría. Y no olvidemos que la colonialidad que ha sostenido a la modernidad, ha significado, entre otras cosas, la negación e invisibilización de muchos saberes que habría que rescatar (Quijano, 2000).

Poco a poco va abriéndose el paso a una psicología más compleja, más rica, más real, que las visiones simplistas, unívocas con las que se han abordado las realidades psicológicas de pueblos y culturas de sabidurías diferentes. Estudios en la India han demostrado la simpleza de querer entender esas realidades desde dicotomías pobres como las del individualismo-colectivismo (Raval y Kral, 2004: 186), o ideas sobre el yo ideal como un individuo autónomo, cuando en el sur de la India es, por ejemplo, el de una persona ligada a un grupo, lo que permite superar diferencias y lograr moderación, equilibrio y complementariedad en las relaciones familiares (Sriram y Chaudhary, 2004:126) y en la interacción afectiva. La lectura y entendimiento de libros como el "Bhagavad-Gita" permitiría tener un conocimiento bastante más rico y más real de la psicología de las personas de la India (A.C. Bhaktivedanta Swami Prabhupada, 1997) y problematizaría "la superstición" de que nuestro conocimiento es universal (Grosfoguel, 2007).

Lo mismo puede aducirse en relación a la comprensión de fenómenos psicológicos en poblaciones de cultura china cuando se quieren aplicar categorías occidentales como culpa y vergüenza. La dificultad surge de "la forma en que términos dicotomizados se generan, prueban y aplican sin bases teóricas, conceptuales y empíricas para entender la estructura profunda de emociones en mundos relacionales culturalmente constituidos" (Hong, 2004: 62). Académicos chinos contemporáneos afirman que el confucionismo es la filosofía más influyente para los actos del pueblo chino común en sus contextos cotidianos (Hong, 2004: 56; Confucius, 1979). Y no se trata de avalar cualquier enfoque sobre el ser humano, sino de abrir una relación de dialogo e interpelación recíproca que fue cerrado con la llamada modernidad.

Sabemos poco sobre la psicología de la cultura árabe porque el auge de esa cultura tuvo lugar entre los siglos VII y IX y el referente clave es sin duda el Corán. En esos siglos crearon una civilización floreciente y un imperio desde el Indo hasta los Pirineos. Pero desde el siglo décimo el pueblo del Profeta fue perdiendo el control de su destino porque sus dirigentes ya no eran árabes y no habían logrado crear instituciones estables. Sin embargo, en la época de las Cruzadas el Mundo Árabe era todavía el depositario de la civilización más avanzada del planeta, intelectual y materialmente, desde Irak hasta la Península Ibérica. La civilización griega llegó a Occidente a través de traductores árabes. En libros árabes quedaron impresos conocimientos que se desarrollaron en medicina, astronomía, química, geografía, matemáticas, arquitectura, agricultura. Entre los siglos XIII y XXI el mundo musulmán ha sido víctima de perpetuas agresiones que hace que el Oriente Árabe sigue viendo en Occidente a un enemigo natural. Hago esta síntesis histórica para señalar que hay una sabiduría musulmana que no hemos valorado y que ayudaría a entender aspectos psicológicos y sociales de mucha actualidad (Cf. Maalouf, 2012: 390-397).

En septiembre de 2107, como ya recordé, tuvo lugar en Durban, Sudáfrica, el primer Congreso Panafricano de Psicología. Habrá que estar muy atentos para aprender de la sabiduría de las culturas africanas en relación a mente y conducta. Es una gran riqueza humana que no solo no hemos valorado suficientemente, sino que hemos ocultado y negado en nombre de la razón universal 
occidental. ¿Qué tienen que decirnos a los psicólogos, por ejemplo, la sabiduría del naturismo Bantú, la sabiduría del animismo centroafricano, la sabiduría del espiritismo egipcio?

Las Culturas Amerindias de este continente que hemos llamado indígenas, originarias, originales, son también fuentes de sabiduría que poco conocemos y que sin embargo configuran las formas de percibir y de concebir la mente y la conducta que subyacen en nuestras supuestas percepciones y concepciones científicas. Cuando hablamos del "naturismo" de los "nativos norteamericanos" sólo nos referimos a visiones simplistas y empobrecedoras de su concepción del ser humano y del mundo. Lo mismo puede decirse de las Culturas Mesoamericanas de las que empezamos a descubrir muchas riquezas, porque las habíamos invisibilizado y ahora buscan reivindicarse con fuerza (Garibay, 1975; Le Clézio, 1988; La Comisión Sexta del EZLN, 2015). En el libro "Panorama literario de los Pueblos Nahuas" Ángel María Garibay ofrece en el capítulo séptimo "La producción didáctica" (138-156), un conjunto de textos que podían servir para ir configurando una psicología del desarrollo humano. Esto mismo está patente en la narrativa de los nahuas contemporáneos de la Sierra Norte de Puebla (Taller de Tradición Oral, 1994). El libro de J.M.G. Le Clézio "Le rêve mexicain ou la pensée interrompue" es una síntesis del aporte posible al pensamiento de nuestros días para enfrentar la crisis civilizatoria en la que estamos inmersos, es un aporte, dice él, que la magia y la luz de los pueblos originarios de México y de América Latina guardan y mantienen vivas. Aportes valiosos para la comprensión del universo y de la realidad de la mente y del comportamiento humano se encuentran también en las culturas indígenas de América del Sur. Esto aparece documentado en el libro de Javier Mendoza Pizarro "El espejo Aymara" publicado en Bolivia en 2015. Se trata de aportes y sabidurías que hay que, por supuesto, problematizar, pero sobre todo que debemos dejar que nos problematicen a los que nos consideramos especialistas en la "ciencia de la psique humana". Ver también por ejemplo el libro "Logos guaraní" de Andino (2019).

\section{Los orígenes de la psicología para el individuo}

Me voy a referir ahora al cuerpo de saberes sobre mente y conducta a partir de las revoluciones de los siglos XVII y XVIII en Occidente. El proceso de la Edad Moderna ha sido el de la creación del individuo como el actor principal en la evolución histórica. Poco a poco se fue emancipando de los condicionamientos colectivos de familia, naturaleza, comunidad y sociedad, bajo el impulso de los pensadores liberales, sobre todo franceses: Rousseau, Voltaire, Diderot, Robespierre, etc. Acontecimientos clave fueron la Declaración de independencia de Estados Unidos en 1776 que culminó en 1783 con la derrota de Inglaterra. Al mismo tiempo tenía lugar la Revolución Industrial gracias al empleo del vapor como nueva fuente de energía. La Revolución Francesa marcó el comienzo político de la Edad Moderna y de la sociedad burguesa. Esos tres acontecimientos históricos marcaron el cambio que resultó en la posibilidad de la emancipación del individuo de su dependencia de la religión, de su dependencia del trabajo agrícola colectivo, de su dependencia de las monarquías. La utopía revolucionaria liberal despertó muchas esperanzas de una época que se caracterizaría por la propuesta francesa de libertad, igualdad y fraternidad. Lo que de hecho aconteció fue el cambio del poder político y económico de la aristocracia a la burguesía, a una modernidad sin fraternidad y a la búsqueda no satisfecha de libertad y de igualdad que generó en 1848 revoluciones en toda Europa (Grosfoguel, 2007).

El cuerpo de saberes burgueses en un mundo "emancipado" fue el de lo razonable (la racionalidad), lo comprobable (el cientificismo), lo confortable (el progreso) y el sinsentido (el desencantamiento). Este aburguesamiento descuidó lo comunal y lo solidario a favor del poder, la riqueza y el prestigio. La humanización del mundo sufrió por la ausencia de la intuicionalidad frente a la racionalidad, por la minusvaloración de la sabiduría frente a la ciencia, por el predominio de la eficacia a costa de la vitalidad, a una vida cotidiana en la que quedó pendiente el asombro. Emancipación y razón se orientaron a la ruptura de vínculos no solamente de opresión, se orientaron a un "progreso" lineal y cuantitativo que reprimió los diferentes registros de la vida humana. Por 
ejemplo, la ruptura con la naturaleza a través de la tecnología - independencia necesaria - desembocó, a partir de la razón instrumental y negadora de límites, en la destrucción del hábitat humano.

\section{Un humanismo comunal y solidario. Las aportaciones escondidas e invisibilizadas}

Si realmente quisiéramos una psicología para el "buen vivir" (Walsh, 2002; Mamani, 2007; Morales, 2018) de todos los habitantes del continente tenemos que empezar por reconocer la diversalidad (la diversidad que reconoce diferencias paro sin establecer jerarquías) de nuestra región. No un multilculturalismo que lo que ha hecho ha sido refuncionalizar la diferencia manteniendo intacta o casi intacta la jerarquía racista/clasista. No podemos abordar simplistamente la realidad, la mente y el comportamiento de las poblaciones de todos los países del continente. Por lo menos deberíamos no perder de vista la gran diferencia de contextos y circunstancias entre los pueblos de Nuestra América y la de los Estados Unidos y Canadá. Y tener claro que las relaciones entre ellos no solo son diferentes o asimétricas. Han sido básicamente dañinas para la mayoría de la población. Planteado esto es preciso enfocar nuestros afanes hacia otros horizontes, hacia un mundo de lo común y de lo solidario, sin caer en romanticismos desatinados, pero en contra de la obsesión del lucro y el mito del éxito. Podríamos desde nuestras especialidades, y en interacción con otras especialidades, contribuir a combatir la depredación delirante del capitalismo: arrasar con suelos, aire, agua y gente. Podríamos hacerlo "sin más ambición que seguir cultivando y tripulando el planeta en su viaje sideral durante el tiempo vital en el que a cada generación le es dado para el cuidado y utilización de la tierra" (Umbral, 2012).

J.M.G. Le Clézio (1988: 243-248) se preguntaba al reflexionar sobre lo que él llamó el pensamiento interrumpido de la Amerindia: ¿Cómo hubieran evolucionado esas civilizaciones, esas religiones si no hubiese tenido lugar la destrucción de la Conquista? ¿Qué filosofía se pudo haber desarrollado? ¿De qué riqueza se nos privó? Le Clézio da por sentado que las creencias, el arte, las virtudes morales de estos pueblos desaparecieron, pero no es así (Taller de Tradición Oral, 1994) como podemos afirmar al haber convivido con descendientes de los nahuas durante 14 años (Sánchez y Almeida, 2005). Aún pueden provocar un cambio profundo en nuestra civilización materialista y oportunista. No hay que olvidar que en el siglo XVI el México indígena estaba más avanzado que Europa en medicina, astronomía, irrigación, drenaje y urbanismo. Lo más valioso y que ha subsistido y que tiene para nuestros tiempos un valor vital es su experiencia de interconexión entre los seres humanos y con el cosmos. Durante mucho tiempo esto pareció haber desaparecido y el indígena quedó sujeto a la pobreza y al desprecio. Pero no es así: creencias y actitudes ancestrales siguen vigentes y podemos hacerlas nuestras y mejorar al mundo. Frente a la violencia desbordada que padecemos podemos escuchar la invitación de los zapatistas "a ver el tiempo que viene, que nos recuerdan que la resistencia no tiene frontera y que lo que necesitamos es resistencia organizada, necio empeño y horizonte" (Gómez, 2017:16).

\section{Pistas de Acción y reflexión desde nuestra praxis (Almeida, en prensa)}

Proponemos pistas para crear bases estructurales de fortaleza, el valor de verdad en las luchas por la dignidad personal y colectiva, y la audacia para crear núcleos cohesivos de amistad y de apoyo.

La propuesta es la Psicología Social Comunitaria Crítica como una ciencia y una filosofía abierta, interactiva, transdisciplinar... Y por lo mismo contrahegemónica, en movimiento, involucrante, peregrinante, más allá del progreso y la revolución.

Su lugar ontológico es la realidad social comunitaria como relatividad, sujeta al devenir, atenta a los desgarramientos sociales, en lo negativo y en lo positivo, en lo disruptivo y lo reconfigurativo.

Su lugar epistemológico es el conocimiento de lo social comunitario como forma de conciencia sujeta al acontecimiento, relativa, consciente de los impactos de los desgarramientos sociales en lo negativo y en lo positivo, en lo disruptivo y lo reconfigurativo.

Los rasgos distintivos de esta Psicología Social Comunitaria Crítica son: 
- Es una praxis que valora más a la gente que a la ciencia hegemónica.

- Es un estudio de la realidad en proceso continuo de emergencia, anclada en lo territorial o itinerante o virtual.

- Se vale de conceptos en perspectiva sociocultural con posibilidades de geopolítica emergente inédita.

- Se opone a las relaciones sociales actuales de racionalidad y consumo con efectos de dominación.

- Considera los condicionamientos biológicos de lo humano dentro de lo cultural y colectivo.

- Aborda la realidad desde los sentipensamientos como formas de conciencia táctiles, proximales, lentas antes que visuales, distales y rápidas, como formas inéditas de abordar el resentimiento y la venganza.

- Es una praxis cultural que abre la puerta a discursos, saberes y prácticas excluidas del discurso científico occidental, abierta a las incertidumbres.

- Es una praxis interpretativa más que un conjunto de saberes y de normas a aplicar, atenta a las subjetividades e intersubjetividades.

\section{¿En dónde estamos?}

En un mundo sin sosiego (Hobsbawm, 2010). A nivel mundial, nacional y local vivimos en la incertidumbre. Incluso regiones que se sentían no tan inseguras como la Unión Europea o los Estados Unidos están experimentando este desasosiego. La economía y la política son fuentes permanentes de inseguridad. En México y América Latina los intereses de unos cuantos mantienen a la mayoría de la población en la miseria.

La crisis mundial del capitalismo sigue haciendo estragos entre las poblaciones más desamparadas. No es posible que 30 millones de mexicanos padezcan de hambre cotidiana. El problema de la pobreza es más que eso, es el empobrecimiento que avanza cada día por la forma como se construye la riqueza. México y América Latina hemos sido una región creada para sostener intereses geopolíticos de otras regiones.

Los terrorismos son la consecuencia natural de grupos de poder que sólo buscan mantener la hegemonía mundial y para ello recurren al crimen organizado y a disponer de una gran población sobrante de miserables como candidatos a ser los soportes humanos del terror.

Por todo el mundo priva entre los grupos privilegiados un elitismo que se desentiende del trágico destino de sus connacionales y que se perpetúa a base de corrupción e impunidad en países en los que las leyes son inoperantes.

En muchas partes del mundo brotan fundamentalismos como reacción ante el azoro y la impotencia que viven muchas poblaciones.

El panorama mundial es de una crisis civilizatoria de proporciones inéditas (Taibo, 2017): incertidumbre, empobrecimiento, terrorismos, elitismos, fundamentalismos, son rasgos comunes en todo el mundo, pero afectan sin duda en mayor grado a las poblaciones de Nuestra América. ¿Tiene la psicología como ciencia y cómo práctica tareas que le conciernen y que no son atendidas? ¿Qué se puede esperar de los psicólogos, de su formación, y de sus conocimientos y habilidades sobre esta crisis mundial? ¿Pueden seguir operando con sus paradigmas habituales? ¿Qué podemos hacer frente al desencanto como estado anímico? ¿Cómo enfrentar la pérdida de referentes ante el trastrocamiento de las dimensiones espaciales y temporales de la existencia? ¿Cómo abordar la gran confusión y desorientación de nuestros procesos conductuales y mentales? No es difícil documentar en lo que acontece en nuestras vidas cotidianas situaciones de espanto que no pueden dejar de inquietarnos.

¿Hacia dónde vamos? Como expresa Pablo Fernández Christlieb (2009): “estamos cansados de ir corriendo a quien sabe dónde en el carro de la postmodernidad, en la autopista de la información, en el tren del progreso, en la ruta del éxito". La postmodernidad nos ha privado de grandes relatos y de motivación. El exceso de información está generando una ausencia de conocimiento y de 
capacidad analítica. El tren del progreso ha significado empobrecimiento para las mayorías, cambio climático y riesgo de extinción de la humanidad. La búsqueda afanosa del éxito ha conllevado la ausencia de solidaridad, ha fomentado la competitividad individual llevada al extremo y a un terrible agotamiento en lo laboral por sobrecargas de todo tipo.

¿Cuáles son nuestras posibilidades cómo psicólogos de contribuir a generar una vida digna para todos nosotros los seres humanos?

Tenemos a nuestro alcance oportunidades de actos de resistencia, de búsqueda y de fraternidad/sororidad. Podemos poner en acción ejercicios de solidaridad en oposición a los egoísmos que genera la modernidad/colonialidad en sus manifestaciones actuales. Podemos estar más atentos a las potencialidades de los indígenas, las mujeres, los homosexuales, los discapacitados, los excluidos, en sus comunidades y en sus agrupamientos de resistencia. No se ha perdido la capacidad de oponerse a condiciones de daño. Es posible detectar formas de combate a la opresión en todas sus formas e instituciones. No se ha perdido la posibilidad de vida en diferencia, en diversalidad: los feminismos descoloniales, las autodefensas comunitarias, las respuestas afrodescendientes a siglos de invisibilización. Está por descubrirse y desarrollarse a niveles nacionales y regionales la fuerza de la no violencia. Todavía disponemos de una gran diversidad de conocimientos ancestrales sobre el valor de la tierra, del agua, del aire y del espíritu. La conservación de la biodiversidad es tarea pendiente para las Ciencias de la Salud y entre ellas para la psicología ambientalista. En todo lo anterior es clave un paradigma que vaya más allá de lo individual y de lo estatal.

En suma, como psicólogos, como practicantes de un campo del saber donde convergen lo biológico y lo sociológico nos corresponde, desde nuestras trincheras, estar atentos a las injusticias y a los agravios que han producido a la humanidad, el patriarcado, el colonialismo y el capitalismo. Nos corresponde desarrollar una psicología menos centrada en el diagnóstico y en la patologización con un paradigma individualista, una psicología más fecunda en la observación de procesos de la vida real. Podemos estar atentos a las oportunidades del "buen ser", de la "buena vida", del "buen vivir" que están surgiendo con los cambios de época y que están siendo fecundadas por muchos seres humanos que mantienen la esperanza de tiempos en los que se hace posible la resistencia a la crueldad del mundo. 


\section{Referencias}

Almeida, E. (en prensa). Los Derechos Humanos, la Violencia Institucional y la Acción Social en México (Diálogo Principal). Santiago de Chile, Chile: 7CIPC.

Almeida y Sánchez (2014). Comunidad: Interacción, Conflicto y Utopía. Puebla, México: UIAP, BUAP e ITESO.

American Psychological Association (2019). Racial Trauma: Theory, Research, and Healing. Special issue of American Psychologist, 74 (1).

Andino, C. (2019). Logos guaraní. Apuntes de pensamiento ético-político paraguayo. Asunción, Paraguay: CEADUC.

Bauvoir, S. de (2013). El segundo sexo. $9^{\text {a }}$ Ed. Buenos Aires, Argentina: DeBolsillo.

Bhaktivedanta Swami Prabhupada, A.C. (1987). El Bhabavad-Gita tal como es. Buenos Aires, Argentina: Fundación Bhaktivedanta.

CIP (2017). XXVI Congreso Interamericano de Psicología. Julio 22 al 27.

Confucius (1979). The Analects. Harmondsworth, U.K: Penguin Classics.

Fernández, Christlieb, P. (2009). La mentalidad de los barcos falsos. En Soto Ramírez, J. Psicologías inútiles (121-140). Ciudad de México, México: UAM Iztapalapa y Porrúa.

Galindo, M. (s.f.). No hay libertad política si no hay libertad sexual. La Paz, Bolivia: Mujeres Creando.

Garibay A.M. (1975). Panorama Literario de los Pueblos Nahuas. Tercera edición. México D.F: Editorial Porrúa.

Gómez, M. (2017). Mensajes para quienes quieran oírlos. Periódico la Jornada, 33, 11753, 16. Martes 18 de abril.

Grosfoguel, R. (2007). Descolonizando los universalismos occidentales en el pluriversalismo transmoderno decolonial de Aimé Césaire hasta los zapatistas. (63-77) En Castro-Gómez, S. y Grosfoguel, R. (Eds.). El giro decolonial: Reflexiones para una diversidad epistémica más allá del capitalismo global. Bogotá, Colombia: Siglo del Hombre Editores.

Hobsbawm, E. (2010). Un mundo sin sosiego. Revista Nexos. 338, 45-53.

Hong, G-Y. (2004). Emotions in Culturally-Constituted Relational Worlds. Culture and Psychology, $10,1,53-63$

IUPsyS (2012). XXX Congreso Internacional de Psicología: “Psychology serving humanity”. 22 al 24 de julio. Capetown, Sudáfrica.

Klein, N. (2014). Capitalism vs the Climate. This changes everything. New York: Simon and Schuster.

La Comisión Sexta del EZLN (2015). Pensamiento crítico frente a la Hidra Capitalista I. Oventic, Chis.: EZLN.

Le Clézio, J.M.G. (1988). Le rêve mexicain ou la pensée interrompue. París, Francia: Gallimard.

Maalouf, A. (2012). Las Cruzadas vistas por los Árabes. 3ra Edición. Madrid, España: Alianza Editorial.

Mamani, C. (2007). Memoria y reconstitución. Intelectuales Indígenas piensan América Latina. Quito, Ecuador: Abya-Yala.

Martín-Baro, I. (1976). Problemas de Psicología Social en América Latina (compilación de textos). San Salvador, El Salvador: UCA.

Martín-Baro, I. (1998). Psicología de la liberación. Madrid, España: Trotta.

Mendoza, Pizarro, J. (2015). El espejo Aymara. Ilusiones ideológicas en Bolivia. La Paz, Bolivia: Plural editores.

Millán, M. (2014). Más allá del feminismo: Caminos para andar. Ciudad de México, México: Red de Feminismos Descoloniales. 
Morales, R. (2018). Comunidad y comunismo: equívocos, confluencias y coincidencias. Perspectivas desde el pueblo mapuche en Chile. En Rozas, G. Decolonialidad, desde la Psicología Social Comunitaria (271- 300). Santiago de Chile, Chile: Universidad Austral de Chile.

Morín, E. (2000). A propos des sept savoirs. París, Francia: Editions Pleins Feux.

OCDE (2019). El Futuro de la Educación en México. Promoviendo Calidad y Equidad. París, Francia : OECD Publishing.

PAPU (2017). 1st PanAfrican Congress of Psychology. Durban, South Africa. Call for abstracts. Psychology for Society. Durban: Author.

Pisano, M. (2001). El triunfo de la masculinidad. Santiago de Chile, Chile: Surada.

Quijano, A. (2000). Colonialidad del poder, eurocentrismo y América Latina. En Lander, E. (comp.). La colonialidad del saber: Eurocentrismo y ciencias sociales. Perspectivas latinoamericanas. Buenos Aires, Argentina: Clacso. (201-246).

Raval, V.V and Kral, M.J. (2004). Core versus Periphery: Dynamics of Personhood over the LifeCourse for a Gujarati Hindi Woman. Culture and Psychology, 10, 2, 162 -194.

Rodríguez, I. (2014). Raíces y tradiciones de la Psicología Social en México. Un estudio historiográfico. Tlaquepaque, México: ITESO, Universidad de Guadalajara, El Colegio de Michoacán.

Rozas, G. (2018). Decolonialidad desde la Psicología Social Comunitaria. Santiago de Chile, Chile: Universidad Austral de Chile.

Sánchez, M.E. (2015). Presentación y comentarios del libro "Más allá del feminismo: Caminos para andar”. Revista de Estudios Latinoamericanos Nueva Época, (35), 168-173.

Sánchez, M.E. y Almeida, E. (2005). Las veredas de la incertidumbre. Relaciones interculturales y supervivencia digna. Puebla, México: UIA Puebla, UAS, UASLP, UV, UJAT, COLPUE, ELPAC, CNEIP.

Sriram, S. and Chaudhary, N. (2004). An Ethnology of Love in a Tamil Family. Margaret Trawick's Notes on Love in a Tamil Family. Culture and Psychology, 10, 1, 11-127.

Taibo, C. (2017). Colapso: Capitalismo terminal, transición ecosocial, ecofascismo. Buenos Aires, Argentina: Libros de Anarres.

Taller de Tradición Oral (1994). Les oíamos contar a nuestros abuelos. México D.F.: INAH.

Umbral (2011). Vigilantes de la tierra. En Ojarasca, 165,2, enero. (Suplemento del periódico La Jornada).

Walsh, C. (2002). De construir la interculturalidad. Consideraciones críticas de la política, la colonialidad, y los movimientos indígenas y negros en el Ecuador. En Fuller, N. (ed.). Interculturalidad y política. 115-142. Lima, Perú: Desafíos Ciencias Sociales en el Perú. 